\title{
Complete Path Planning for Planar Closed Chains Among Point Obstacles
}

\author{
G.F. Liu J.C. Trinkle \\ Department of Computer Science \\ Rensselaer Polytechnic Institute \\ Troy, New York 12180 \\ Email: liugf,trink@cs.rpi.edu
}

\begin{abstract}
A method to compute an exact cell decomposition and corresponding connectivity graph of the configuration space (C-space) of a planar closed chain manipulator moving among point obstacles is developed. By studying the global properties of the loop closure and collision constraint set, a cylindrical decomposition of the collision-free portion of $\mathrm{C}$-space (C-free) is obtained without translating the constraints into polynomials as required by Collins' method [1]. Once the graph is constructed, motion planning proceeds in the usual way; graph search followed by path construction. Experimental results demonstrate the effectiveness of the algorithm.
\end{abstract}

\section{INTRODUCTION}

Because of their speed and stiffness, parallel manipulators have recently attracted the interest of robotics researchers and industrial users. However, their closed loop structure gives rise to joint variable dependencies, which manifest in a topologically complex configuration space. An important consequence of this is that, in general, the C-space cannot be globally (and smoothly) parameterized by a single set of $d$ variables (for example a subset of the joint displacements), where $d$ is the number of degrees of freedom of the manipulator. In other words, any $d$-dimensional atlas of $\mathrm{C}$-space will contain multiple charts. This fact generally makes motion planning more challenging for parallel manipulators than it is for serial manipulators.

\section{A. Previous Work}

It is well known that general exact motion planning algorithms for serial manipulators are highly complex [5], [6], [7], [8], [16]. In fact, the most efficient exact planning algorithm is Canny's, whose worst-case time complexity is exponential in the dimension of C-space [6]. In principle, exact algorithms can be applied to systems with holonomic equality constraints such as those imposed by the closed kinematic loops in parallel manipulators "by defining convenient charts and managing them" (see [7], page 411). However, the difficulty of implementing exact algorithms for general systems fueled a paradigm shift to sample-based algorithms [9], [10], [11].

Sample-based algorithms build a graph that approximates the global structure of the collision-free portion of C-space (C-free). The graph has nodes that correspond to selected points of $\mathrm{C}$-free and arcs between nodes that indicate path connectedness between the corresponding points. The graph can be thought of a network of highways, or a roadmap, of
C-free. The roadmap becomes suitable for motion planning when the following two attributes are attained: (1) there is a one-to-one correspondence between components of the graph and components of $\mathrm{C}$-free, and (2) given a point in $\mathrm{C}$-free, it is easy to find a path connecting it to the graph. At this point, motion planning is essentially reduced to graph searching.

Sample-based algorithms have been quite successful for systems whose $\mathrm{C}$-space can be parameterized by a single chart with number of coordinates equal to the number of degrees of freedom of the system, but less successful otherwise [12], [17]. Even though one can always generate an ambient space parameterizable by a single chart by choosing more parameters than the dimension of $\mathrm{C}$-space, the number of sample points needed to construct a good roadmap grows exponentially with the dimension of the ambient space, because the number of connected components of the collision-free portion of this space grows exponentially with its dimension. Second, for most parallel manipulators of interest, parameterization of $\mathrm{C}$-space using the minimal number of coordinates requires multiple charts. These can be difficult to define and to choose suitable metrics to obtain globally "well distributed" sample points. Although the RLG method [13] provides partial remedies to traditional sample-based algorithms by estimating the motion range of each joint of the linkage that obeys the loop closure constraint, the algorithm fails to sample the boundary and narrow passages, and thus may give a wrong answer for some path queries.

The difficulties associated with applying sample-based motion planning methods to parallel manipulators and the availability of new results in topology led to renewed interest in exact planning algorithms for closed kinematic chains (see Figure 1) [18], [19], [20]. Trinkle and Milgram derived some global topological properties of the $\mathrm{C}$-space (the number of components and the structures of the components) of singleloop closed chains with spherical joints in a workspace without obstacles [18]. These properties drove the design of a complete motion planning algorithm that works roughly as follows.

1) Choose a subset $\mathcal{A}$ of the links that can be positioned arbitrarily, and yet the remaining links can close the loop;

2) Move the links in $\mathcal{A}$ to their goal orientations along an arbitrary path while maintaining loop closure;

3) Permanently fix the orientations of the links in $\mathcal{A}$; 
4) Repeat until all links are fixed.

The main result that guided the algorithm's design is Theorem 2 in [18]. In essence, the C-space is the union of manifolds that are products of spheres and intervals. The joint coordinates corresponding to the spheres are those that can contribute to the subset $\mathcal{A}$ mentioned above and the structure of the $\mathrm{C}$-space suggests a local parameterization (i.e., "convenient chart") for each step.

The planning algorithm for closed chains in [18] was not designed to handle obstacles. In [19], [20], point obstacles were added to the workspace of a planar manipulator, but the closed chain constraint was relaxed. The portion of C-space corresponding to collisions between the manipulator and the obstacles (the C-obstacle) was analyzed in detail, to reveal that C-free locally fibres over a lower-dimensional base manifold with fibers composed of open intervals (called local component sheaves). "Gluing" together the sheaves produces cells of Cfree, whose boundaries are determined by their critical points.

\section{B. Contribution}

In this paper, the concepts used in [18], [19], [20] are brought together to design an algorithm to construct an exact cell-decomposition of C-free of an $m$-link planar closed chain moving among point obstacles. The main steps are:

1) Partition $\mathrm{C}$-space into two pieces embedded in two ( $\mathrm{m}$ 3)-dimensional tori;

2) Compute the boundary of the loop closure constraint variety to identify the reachable portions of $\mathrm{C}$-space;

3) Compute the collision varieties in each torus and construct a connectivity graph of $\mathrm{C}$-free while ignoring the loop closure constraint;

4) Use the boundary of the loop closure variety to refine the graphs and to determine their connectivity.

The key step of our approach is Step 2 since boundary variety plays an important role in determining the global connectivity of C-space as well as identifying its reachable portions. In our approach, the structure of the boundary is obtained via the C-spaces of a set of recursively constructed planar closed chains.

As a byproduct of the approach, it is trivial to determine cell membership and reachability of an arbitrary point in Cspace. Given this fact and the graph, one can easily test motion planning problems for path existence and then construct a path if one exists.

\section{BASIC NOTATION AND TERMINOLOGY}

Imagine a planar serial chain of $m-1$ links connected by revolute joints, with one end free, and the other connected to the ground. The ground is regarded as link $m$ and is referred to as the base of the chain. Relative to the base, the open chain has $m-1$ degrees of freedom and its C-space is simply a product of $m-1$ circles, (i.e., $\left.\mathcal{C}=\left(S^{1}\right)^{m-1}\right)$. A closed $m$ chain can be constructed by attaching the distal end of the open chain to the base as shown in Figure 1. Mathematically, this attachment imposes two algebraic equality constraints, causing the C-space of the closed chain to become a compact, closed,

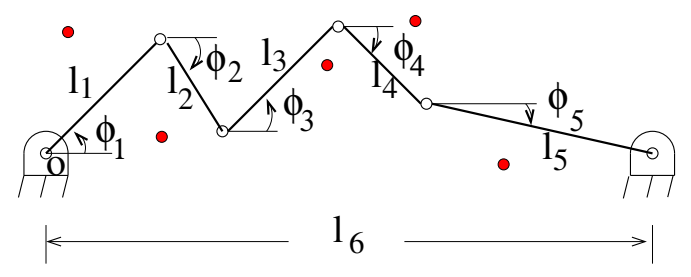

Fig. 1. A closed 6-chain among point obstacles (shown as small discs).

real, variety of dimension $m-3$. This variety is a manifold as long as the distance between the two base connections is not equal to one of the $2^{m-2}$ critical lengths [18].

To fix notation, let $\left\{l_{1}, \cdots, l_{m}\right\}$ denote the fixed link lengths and $\left\{\phi_{1}, \cdots, \phi_{m}\right\}$ denote their angles measured counterclockwise from the vector from the center of joint 1 to the center of joint $m$. Since our interest is in motions of the closed chain relative to the base, $\phi_{m}$ is set to zero.

Suppose that there is a finite set $\mathcal{O}$ of point obstacles $\left\{p_{1}, \cdots, p_{n}\right\}$ that the closed $m$-chain may not touch. The set of configurations for which a link intersects a point obstacle forms an arrangement of $(m-4)$-dimensional varieties. The union of these varieties is the $\mathrm{C}$-obstacle $\mathcal{C}_{\text {obst }}$. If link-link collisions are also to be avoided, $\mathcal{C}_{\text {obst }}$ becomes the union of the (m-4)-dimensional collision varieties and an ( $m-3)$ dimensional link-link collision set. $\mathrm{C}$-free, denoted by $\mathcal{C}_{\text {free }}$, is the compliment of $\mathcal{C}_{\text {obst }}$ in $\mathcal{C}$.

Finally, the path planning problem can be stated as follows: given $\phi_{\text {init }}=\left(\phi_{1}, \cdots, \phi_{m}\right)_{\text {init }} \in \mathcal{C}_{\text {free }}$ and $\phi_{\text {final }}=$ $\left(\phi_{1}, \cdots, \phi_{m}\right)_{\text {final }} \in \mathcal{C}_{\text {free }}$ determine a continuous map $\tau \in$ $[0,1] \mapsto\left(\phi_{1}(\tau), \cdots, \phi_{m}(\tau)\right) \in \mathcal{C}_{\text {free }}$ such that $\phi(0)=\phi_{\text {init }}$ and $\phi(1)=\phi_{\text {final }}$, or report that no such path exists.

\section{C-SPace of Planar Closed Chains}

Here we summarize three results from topological approaches to motion planning that were crucial to the work presented here. The first result is about the connectivity of the C-space of a planar closed chain. We need a concept called "long links" [18] which is defined as a subset $\mathcal{L}$ of the links such that the sum of the lengths of every pair of distinct links in $\mathcal{L}$ is strictly greater than half of the sum of the lengths of all $m$ links. Due to the strict inequality in the definition, the number of long links $|\mathcal{L}|$ must be 0,2 , or 3 . If $|\mathcal{L}|$ is equal to 3 , the C-space has two components; otherwise, it has one.

The second result gives the topology of the C-space. It says that for given lengths $\left\{l_{1}, \cdots, l_{m-1}\right\}$ and base length $l_{m}$ that is generic with respect to those $m-1$ lengths, the C-space is the boundary of a manifold with boundary, which is given as the union of sub-manifolds of the form $\left(S^{1}\right)^{k} \times I^{m-2-k}$ [18], where $I^{d}$ denotes the interval of dimension $d$.

To clarify the above conclusion, consider Figure 2, which shows a horizontal base link and three moveable links anchored at the center of four concentric circles. These circles are the critical circles (not drawn to scale) of the open 3-chain. If the end point of the 3-chain is anchored at any point in the shaded annular region, its $\mathrm{C}$-space is that of a closed 4-chain. If the anchor point is interior to one of the three reachable 


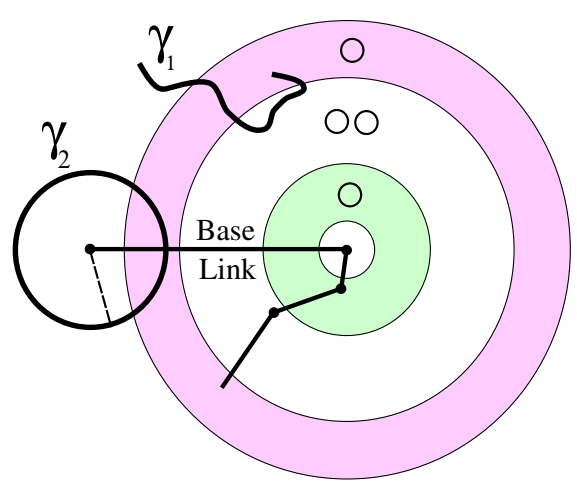

Fig. 2. Construction of C-space of closed chains via critical circles of an open chain.

annuli, that C-space is one circle or two disjoint circles (as indicated by the small circles at the 12 o'clock position of the concentric circles).

Assume that the end point of the open 3-chain is connected to an open chain based at the left end of the base link, and further, assume that as a result of the connection, the end-point of the previously open 3-chain has one degree of freedom, thus effectively creating a closed 5-chain. Two possible workspaces for the end-point of the open 3-chain are shown: the curve segment $\gamma_{1}$ and the circle $\gamma_{2}$.

The C-space of the newly-formed closed chain can be determined by "gluing" together all the C-spaces at each point as we move along $\gamma_{j}$ from one end to the other. For example, begin at the left end of curve $\gamma_{1}$ and traverse it to its other end. Initially, the $\mathrm{C}$-space over each point of $\gamma_{1}$ is empty, since the open 3-chain cannot reach those points. At the intersection with the outer-most circle, the C-space of the closed chain is a point, but the workspace segment lying inside the outer-most annular region generates a tube. At the point where the curve intersects the next critical circle, the C-space of the closed 5-chain is a figure eight. This signifies a bifurcation of the tube into two tubes. The two tubes coalesce into a single tube at the next crossing of the same critical circle. Finally, at the end of the curve, $\mathrm{C}$-space is a circle. Thus the $\mathrm{C}$-space of the mechanism with the end of the open 3-chain constrained to lie on $\gamma_{1}$ is a tube pinched closed at one end, open at the other, and with a hole through the tube somewhere between the two ends. Applying the same logic to the closed 5-chain that would result from connecting the end of the open 3-chain to the link shown as a dashed line, one finds that the $\mathrm{C}$-space of the closed 5-chain is a sphere.

Following this approach, one can show by constructing circles and base links of various sizes that a closed 5-chain has up to six types of C-spaces, among which those shown in Figure 3 are connected. The sixth type of C-space is the disjoint union of two copies of $T^{2}$. Here $S^{2}$ and $T^{2}$ represent the two-dimensional sphere and torus, respectively, and \# denotes the connected sum of two spaces.

The third result pertains to the parametrization of the $\mathrm{C}$ space. Since the $\mathrm{C}$-space of a generic closed $m$-chain is an

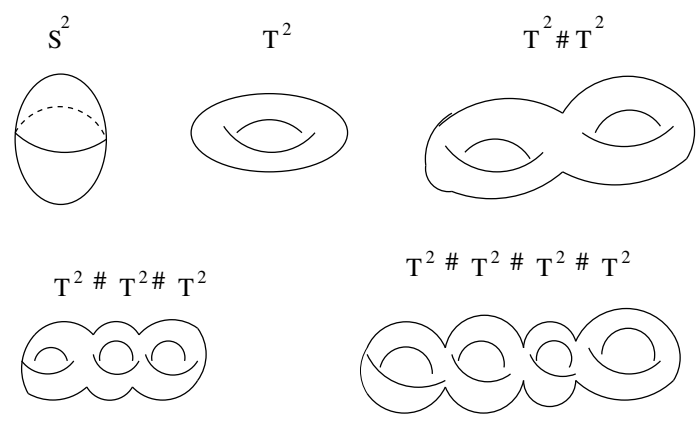

Fig. 3. Five types of connected C-spaces of closed 5-chains.

( $m$-3)-dimensional manifold, C-space can be locally parameterized by a set of $m-3$ joint angles. However, fixing the orientations of $m-3$ links (in addition to the fixed base), does not fix the configuration of the closed chain. Returning to Figure 1, fixing $\phi_{3}, \phi_{4}$ and $\phi_{5}$ still allows elbow-up and elbow-down postures of links 1 and 2 .

This last result suggests partitioning $\mathrm{C}$-space into an elbowup piece and an elbow-down piece as follows. Break the closed chain at the third joint, thus creating an open 2-chain and an open $(m-3)$-chain based at opposite ends of the base link. The $\mathrm{C}$-space of the second open chain is the $(m-3)$-dimensional torus. For an arbitrary point in this space, the chain can be closed in 0,1 , or 2 configurations of the 2-chain. When there are two configurations, they are labeled elbow-up and elbowdown. Since there are never more than two configurations that close the loop, two copies of the torus suffice to represent the C-space of the closed chain. When there is only one configuration, the elbow-up and elbow-down configurations have converged, so at these points, the tori are connected. These configurations form a variety referred to as the boundary variety that is the subject of the next section.

\section{BOUNDARY VARIETY AND ITS DECOMPOSITION}

In this section, we outline a recursive projection method (similar to the approach in [19], [20]) to determine the structure and a cell decomposition of the boundary variety. The $k^{\text {th }}$ level of recursion will be denoted by appending " $(k)$ " to the expression in question. Refer to Figure 1. As described above, let us break the closed $m$-chain into an open 2-chain $\mathrm{CH}_{1}(1)$ with link lengths $\left\{l_{1}, l_{2}\right\}$, and an open (m-3)-chain $\mathrm{CH}_{2}(1)$ with link lengths $\left\{l_{3}, \cdots, l_{m-1}\right\}$ based at the point $\left(l_{m}, 0\right)$. Choose the joint angles of $\mathrm{CH}_{2}(1)$ as the parameterization of the elbow-up and elbow-down tori. Further, let $f(1)$ be the forward kinematic map of $\mathrm{CH}_{2}(1)$.

The boundary variety $B(1)$ is the set of all configurations for which the endpoints of the two open chains can be connected when the links of the open 2-chain are collinear. With the constraint of collinearity, the space of possible end point locations $\Sigma(1)$ of the 2 -chain in the workspace is a pair of concentric circles of radii $l_{1}+l_{2}$ and $\left|l_{1}-l_{2}\right|$ centered at the origin. The boundary variety can now be defined as follows:

$$
B(1)=f(1)^{-1}(\Sigma(1))
$$


Note that $B(1)$ is the union of the $\mathrm{C}$-space of the two closed $(m-1)$-chains $M_{1}(1)$ and $M_{2}(1)$ with link lengths $\left\{l_{1}+l_{2}, l_{3}, \cdots, l_{m}\right\}$ and $\left\{\left|l_{1}-l_{2}\right|, l_{3}, \cdots, l_{m}\right\}$. Also, $B(1)$ is empty if and only if the intersection between $\Sigma(1)$ and the annulus centered at $\left(l_{m}, 0\right)$ with radii $\sum_{i=3}^{m-1} l_{i}$ and $\min \left(\| l_{3} \pm\right.$ $\left.\cdots \pm l_{m-1} \|\right)$ is empty, in which case, $\mathrm{C}$-space is not connected.

The process described above is repeated for each of the two closed $(m-1)$-chains. That is, each of these closed chains is assumed to have their third joints removed, which gives rise to $2^{2}$ critical circles centered on the origin with radii $\mid l_{1} \pm l_{2} \pm$ $l_{3} \mid$ and whose union is $\Sigma(2)$. Similar to $B(1)$, the boundary variety $B(2)$, is given as $B(2)=f(2)^{-1}(\Sigma(2))$, where $f(2)$ is the forward kinematic map of the $\left\{l_{4}, \cdots, l_{m-1}\right\}$ open $(m-$ 4)-chain $\mathrm{CH}_{2}(2)$ based at the point $\left(l_{m}, 0\right)$. By construction, it is clear that $B(2)$ is the union of the $\mathrm{C}$-spaces of the four closed $(m-2)$-chains each with first link length equal to one of the four critical radii. $B(2)$ is also the set of critical values, or skeleton of the projection of $B(1)$ onto the $(m-4)$-dimensional torus with coordinates $\left\{\phi_{4}, \cdots, \phi_{m-1}\right\}$.

Recursion continues until $B(m-3)$ is defined. In this case, $\Sigma(m-3)$ is the union of $2^{m-3}$ concentric circles centered at the origin. The boundary variety $B(m-3)$ is the set of values of $\phi_{m-1}$ where the circle of radius $l_{m-1}$ centered at $\left(l_{m}, 0\right)$ intersects the circles comprising $\Sigma(m-3)$. With $\{B(1), \cdots, B(m-3)\}$ defined, C-space can now be decomposed into reachable and unreachable cells which are $(m-3)$ dimensional cylinders.

Example: Consider a closed 6-chain, with link lengths, $\{0.5512,1.9457,1.2131,2.9482,4.5684,5.7815\}$. The Cspace of this chain is connected, since there are only two long links. It is contained in two three-dimensional tori that are connected through the boundary variety $B(1) . B(1)$ is the union of the C-spaces of two closed 5-chains $M_{1}(1)$ and $M_{2}(1)$ with the last four link lengths $\{1.2131,2.9482,4.5684,5.7815\}$ and the first link of length equal to one of the critical lengths $\{1.3945,2.4969\}$ of the circles composing $\Sigma(1)$. Using the approach described in Figure 2, the C-spaces of each of $M_{1}(1)$ and $M_{2}(1)$ is a bitorus, $T^{2} \# T^{2}$ (see Figure 3 ). This is consistent with the fact that each of of $M_{1}(1)$ and $M_{2}(1)$ have two long links.

To draw $B(1)$, it is convenient to represent a 3-torus as a three-cube with edge length $2 \pi$ and opposite faces identified. In this space, a bitorus (qualitatively like those composing $B(1)$ ) is shown in Figure 4. Note that the bold girth curve and the two circles where the bitorus is cut (recall that the top and bottom circles are identified) is the skeleton of the bitorus under the projection map onto the $\left(\phi_{4}-\phi_{5}\right)$-space.

$B(2)$ is the skeleton of $B(1)$ under the vertical projection onto the $\left(\phi_{4}-\phi_{5}\right)$-face of the cube. In Figures 6 and 7, $B(2)$ is plotted in thin solid closed curves. Note that $B(2)$ consists of six circles; two pairs of small "concentric" circles and one pair of large concentric circles containing the other two pairs. The large circles are the projections of the girth curves of the bitori of $B(1)$. The two pairs of small circles are the small circular skeletal curves like those shown in Figure 4. The fact

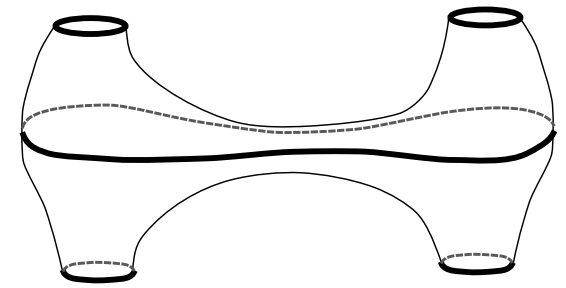

Fig. 4. A bitorus drawn in a cubical represenation of C-space. The skeleton of this bitorus under a vertical projection map (drawn bold), is three circles.

that these pairs of circles are concentric implies that the two bitori of $B(1)$ are "nested" in C-space.

The last step is to project the skeleton of $B(2)$ to an edge of the cube. This is shown by the 12 dashed vertical lines (three are covered by other vertical lines) in Figures 6 and 7. Using the approach discussed in Figure 2, one can show that six of the eight critical circles with the radii $\{6.6582,5.5558,4.2320,3.1296,2.7668,1.6644\}$ are intersected transversally by the workspace of the end point of the open 1-chain $\mathrm{CH}_{2}(3)$. These correspond to the 12 critical values of $\phi_{5}$.

\section{Collision VARIETIES}

Let $V_{p_{i}}^{j}, j=1, \cdots, m-1$, denote the $(m-1)$-dimensional variety corresponding to $p_{i}$ lying on link $j$. The union of these varieties over all links gives the contribution of $p_{i}$ to the Cobstacle:

$$
V_{p_{i}}=\bigcup_{j=1}^{m-1} V_{p_{i}}^{j}
$$

The union of the $V_{p_{i}}$ over all point obstacles in $\mathcal{O}$ is denoted by $V$ :

$$
V=\bigcup_{i=1}^{n} V_{p_{i}}
$$

To study the global structure of these varieties, the closed chain is again broken into the open 2-chain $\mathrm{CH}_{1}(1)$ and the open (m-3)-chain $\mathrm{CH}_{2}(1)$. The contact varieties of $\mathrm{CH}_{2}(1)$ are already understood from previous work [20]. However, our interest is in $V_{p_{i}}^{j}$ for $j=m-1, \cdots, 3$, and $i=1, \cdots, n$ ), which can be viewed as the contact varieties of $\mathrm{CH}_{2}(1)$ clipped by the $B(1)$. The portions of the constraint varieties of $\mathrm{CH}_{2}(1)$ lying on the unreachable side of $B(1)$ are eliminated. Figure 5 shows a cylinder with rectangular cross section in a threedimensional C-space. The cylinder is cut by $V_{p_{1}}, V_{p_{2}}$ and two patches of $B(1)$, labeled $B_{1}(1)$ and $B_{2}(1)$. Assuming the top and bottom of the rectangular column are identified and ignoring $B(1)$, there are two cells in the cylinder. Assuming that the region above the top patch and below the bottom patch of $B(1)$ are unreachable, a portion of $V_{p_{2}}$ is clipped and there are three cells.

The topological properties of the remaining varieties, $V_{p_{i}}^{1}$ and $V_{p_{i}}^{2}, i=\{1, \cdots n\}$, are determined by the technique developed in [18] and described in the discussion of Figure 2. 
These varieties can be expressed as follows:

$$
\begin{aligned}
& V_{p_{i}}^{1}=f(1)^{-1}\left(\gamma_{1}^{i}\right) \\
& V_{p_{i}}^{2}=f(1)^{-1}\left(\gamma_{2}^{i}\right)
\end{aligned}
$$

where $\gamma_{j}^{i}$ is the workspace of the end-point (always a closed loop) of $\mathrm{CH}_{1}(1)$ when link $j$ is in contact with $p_{i}$ and $f(1)$ is the forward kinematic map of $\mathrm{CH}_{2}(1)$. Again it is important to understand the intersection of these contact varieties with $B(1)$. Since the boundary constraint requires links 1 and 2 to be colinear, the intersections of $V_{p_{i}}^{1}$ and $V_{p_{i}}^{2}$ with $B(1)$ can be seen to be the $\mathrm{C}$-spaces of two closed $(m-2)$-chains formed by replacing links 1 and 2 by a single link of length $\left|l_{1} \pm l_{2}\right|$ and fixing this link in contact with $p_{i}$.

Example continued: We introduce two point obstacles, $p_{1}=(4,2)$ and $p_{2}=(3,1)$. Note that it is impossible for link 1 or 2 to touch either point, so the corresponding varieties are empty. Consequently, only the contact varieties of links 3, 4, and 5 appear in Figures 6 and 7. Note that the two thickest vertical lines at $\phi_{5} \approx \pm 1.38$, define extreme points of the boundary variety $B(1)$. By these figures, one can also determine the structure of $V_{p_{i}}^{5}$, which projects to the two second thickest vertical lines in the Figures. Take $V_{p_{2}}^{5}$ as an example. The projection of $V_{p_{2}}^{5}$ is the line $\phi_{5} \approx-0.345$ whose intersection with the interior between the pair of large concentric circles of $B(2)$ is two separated line segments, and with the interior of the inner pair of circles an interval. This reveals that the cross-section of $V_{p_{2}}^{5}$ in the horizontal plane of each 3-torus changes from two separated segments to one segment, and then back to two separated segments (recall that opposite faces of each 3-torus are identified). The boundary of the cross-section is either four or two separated points, the union of which gives $B(1) \bigcap V_{p_{2}}^{5}$. Gluing the two pieces of $V_{p_{2}}^{5}$ in the elbow-up and elbow-down tori along $B(1) \cap V_{p_{2}}^{5}$ yields the surface $T^{2} \# T^{2}$. This result can also be obtained using the approach described in Figure 2 for the corresponding closed 5-chain of $V_{p_{2}}^{5}$ with the link lengths $\{0.5512,1.9457,1.2131,2.9482,2.1415)$, which has two long links. Using the same analysis method, we can see that $V_{p_{1}}^{5}$ is a torus $T^{2}$ (in Figures 6 and 7 , the line of $V_{p_{1}}^{5}$ has no intersection with the interior between the inner pair of circles).

\section{GRAPH REPRESENTATION OF C-FREE}

The recursive decomposition of the elbow-up and elbowdown tori makes the construction of a connectivity graph straight forward. In essence, the graph construction process is recursive, starting with the one-dimensional circle parameterized by $\phi_{m-1}$ and working up to the full $(m-3)$-dimensional C-space. Referring to Figure 6, the circle in $T_{u}^{2}$ parameterized by $\phi_{5}$ has 12 distinct critical points. Removal of these points from the $\phi_{5}$ circle defines a set of 12 open intervals. Some of the two-dimensional cells above these points could be disconnected at these points, but whether or not this is the case is only revealed as the method proceeds. Therefore, initially, these intervals and the cells above them are assumed to be disconnected at the critical points. Thus, at this stage, the graph of the space of $\phi_{m-1}$ is simply 12 disconnected nodes. The same decomposition occurs in $T_{d}^{2}$ as shown in Figure 7 , but no attempt is made to connect the two graphs yet.

The next step is to "lift" the graphs so that they represent the cell structure of the two 2-tori parameterized by $\phi_{m-1}$ and $\phi_{m-2}$. The intervals identified in the first step serve as the base manifolds for the second step. If one fixes $\phi_{m-1}$, then $\phi_{m-2}$ lies on a circle. For the critical values of $\phi_{m-1}$, the circle is drawn as a dashed vertical line ( 9 of the 12 are visible). Between the critical circles are two-dimensional cylindrical cells. For example, consider the two critical circles straddling $\phi_{5}=1$ in Figure 6. This portion of C-space is a or tube that is cut in four places by the projected skeleton of the boundary variety. This identifies four two-dimensional cells. The tube just to the right is cut in only two places, yielding two cells. However, in the $\left(\phi_{m-1}, \phi_{m-2}\right)$-space some of the cells in the two tubes are connected. After merging the connected cells, three possibly disconnected cells remain.

The graphs are again lifted, this time into the $\left(\phi_{m-1}, \phi_{m-2}, \phi_{m-3}\right)$-space. Figure 5 shows a cell in the $\left(\phi_{m-1}-\phi_{m-2}\right)$-space. Above it is the $\left(\phi_{m-1}, \phi_{m-2}, \phi_{m-3}\right)$ space with two-dimensional constraints and boundary varieties. The locus of critical points are those in the intersection of the surfaces. Its projection onto the cell is the curve D, which splits the cell into two. Above each non-critical point in the two cells, there are four constraint surfaces. Assuming that the space above $B_{1}(1)$ and below $B_{2}(1)$ violates the loop closure constraint, then the four surfaces define two reachable cylindrical cells above the cell $(\mathrm{a}, \mathrm{D})$ and three cells above (D,b). Further the two cells bounded below by $B_{2}(1)$ are connected, as are those bounded below by the surface labeled " 2 ." Merging yields three cells in $\left(\phi_{m-1}, \phi_{m-2}, \phi_{m-3}\right)$-space above the original cell in $\left(\phi_{m-1}, \phi_{m-2}\right)$-space.

Analyzing all adjacent cells in $\left(\phi_{m-1}, \phi_{m-2}\right)$-space in the same way yields all connected components of the $\left(\phi_{m-1}, \phi_{m-2}, \phi_{m-3}\right)$-space, and the process continues until all connected components of dimension $m-3$ are identified.

\section{EXPERIMENTAL RESULTS}

Our method for closed 5-chains and 6-chains was implemented in Matlab and tested for many planning problems. Typically, a closed 5-chain moving among 4 point obstacles required about 160 seconds, and a closed 6-chain moving among 2 point obstacles required about 210 seconds.

Example further continued: A connectivity graph of Cfree was constructed according to the approach described above. Then a motion planning problem was specified by the following configurations:

$$
\begin{aligned}
\phi_{\text {init }} & =(-0.6363,-1.2183,0.0416,1.9416,-0.1416,0) \\
\phi_{\text {goal }} & =(-0.9063,0.8648,0.0416,-2.0416,0.3416,0) .
\end{aligned}
$$

One of the configurations was in the elbow-up torus; the other was in the elbow-down torus. The computed path was 


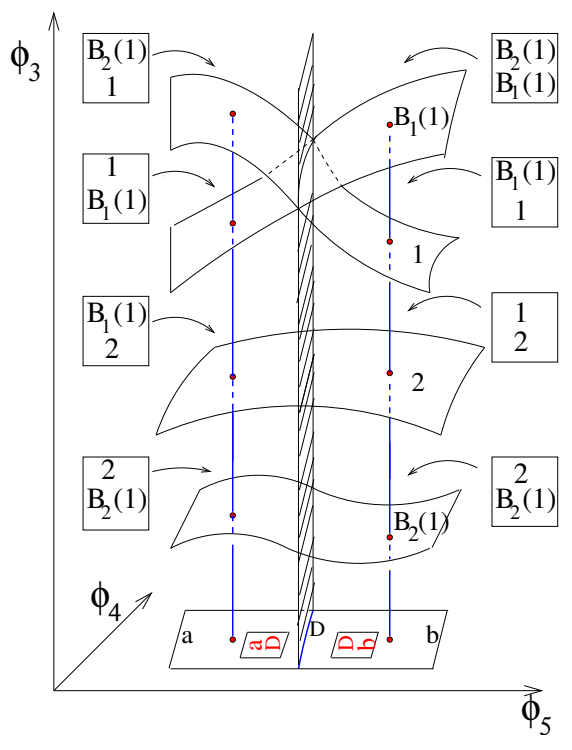

Fig. 5. A rectangular cell in $\left(\phi_{4}, \phi_{5}\right)$-space onto which the critical points of constraint varieties in $\left(\phi_{4}, \phi_{5}, \phi_{6}\right)$-space are projected.

projected onto the two two-dimensional tori shown in Figures 6 and 7. (Note that in these lower-dimensional spaces, one should not expect the path to jump from the elbow-up to the elbow-down spaces through the projection of $B(1)$ ). While it is difficult to see in a three-dimensional plot, the path in the full C-space crosses through the boundary variety $B(1)$. Animation of the motion in this example can be found in ftp: / / 6bar: 6bar@143.89.47.18.

\section{COMPlEXity AnAlysis}

The complexity of any algorithm that performs cell decomposition on $\mathrm{C}$-free is bounded from below by the number of connected components. While we have not proven this, there is evidence that suggests that this lower bound is $\Omega\left(n^{m-3}\right), m \geq$ 5 . We also conjecture an upper bound, by considering the polynomial representation of the collision and loop closure varieties. Assuming that the highest degree of these polynomials is $\delta$, then Halperin's cell complexity results [21] applied to our approach imply that each connected component of Cfree could be composed of $O\left(n^{m-4} \delta\right)$ cells. Since each of these cells must be computed, the worst-case complexity of our decomposition algorithm is $O\left(n^{2 m-7}\right), m \geq 5$.

To provide insight into our conjecture, we show how to obtain these results for the case of $m=5$ with $n$ point obstacles. The $\mathrm{C}$-space is two-dimensional and the contact varieties are one-dimensional curves in C-space. An upper bound on the number of components can be obtained by imposing three conditions: (1) all elbow-up and elbow-down configurations are valid, (2) all contact varieties are closed, and (3) all possible intersections among varieties occur and they occur in general position. These conditions can be satisfied only if:

$$
l_{5}=0, l_{1}=l_{4}, l_{2}=l_{3}, l_{2}>2 l_{1}
$$

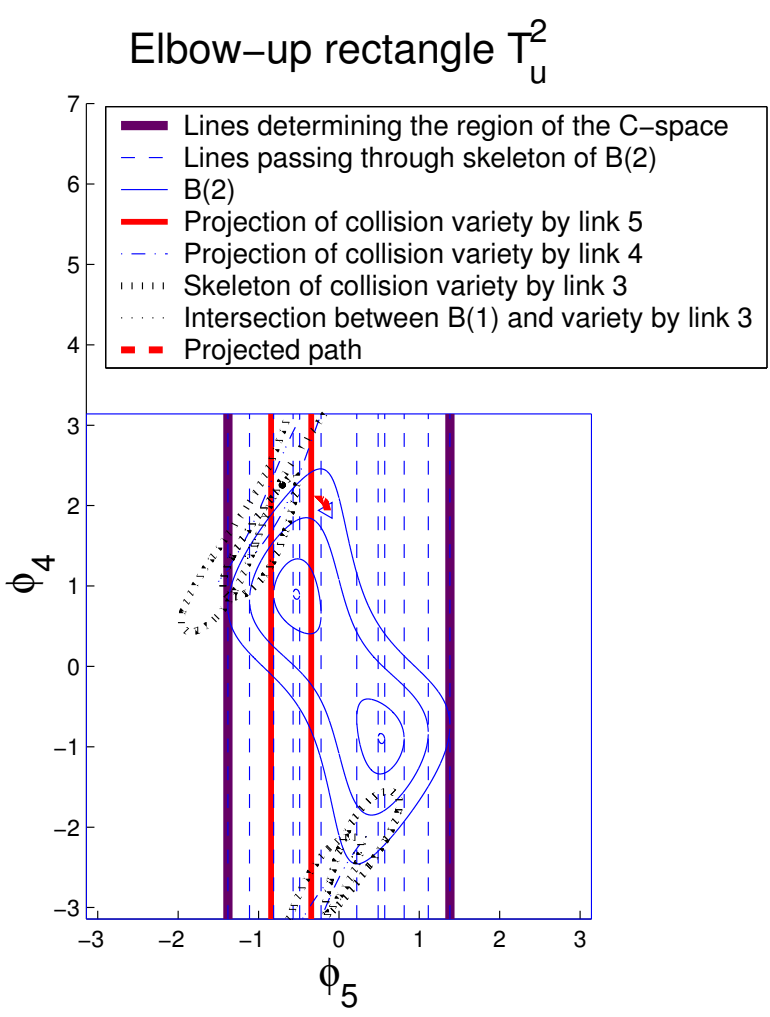

Fig. 6. The path (drawn as red dashed line) projected to $T_{u}^{2}$. The two triangles pointed to the left and right represent the initial and goal configurations, respectively

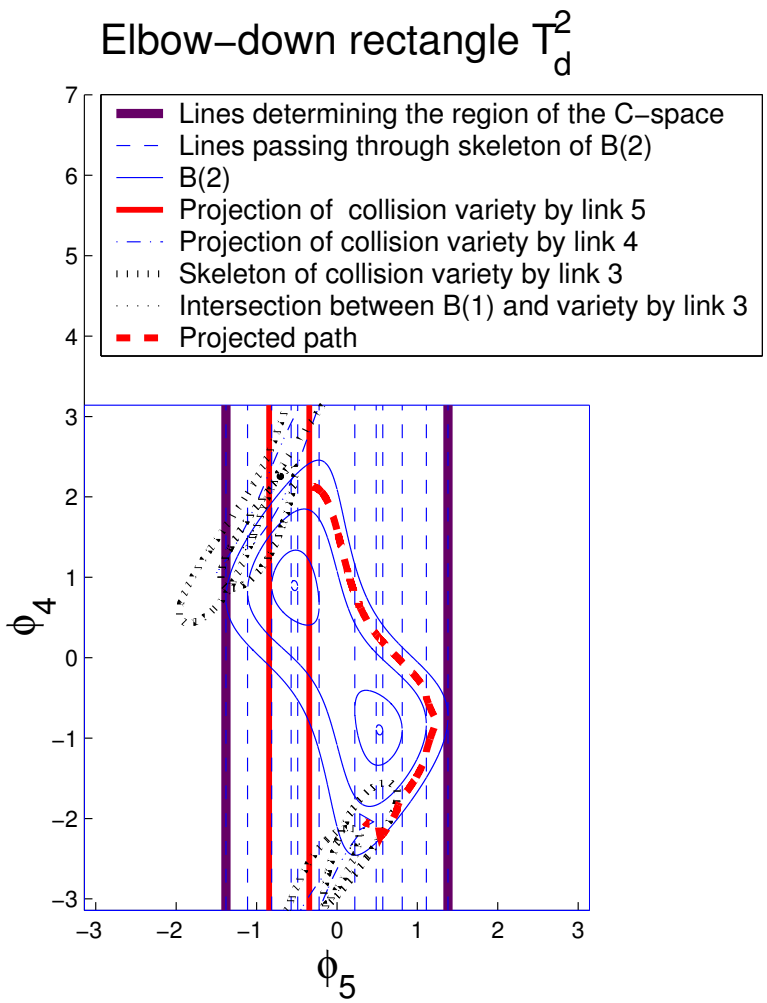

Fig. 7. The path (drawn as red dashed line) projected to $T_{d}^{2}$. 
which defines a degenerate closed chain with base link of length zero. The result is effectively a closed four-chain with base link pinned at one end rather than both. One can see that it is always possible to move $\phi_{4}$ and $\phi_{3}$ arbitrarily and that for every $\phi_{4}$ and $\phi_{3}$ there are two configurations of the other two links; elbow-up and elbow-down. Thus C-space is two copies of $T^{2}, T_{u}^{2}$ and $T_{d}^{2}$, glued along the two circles corresponding to configurations where all the links are colinear.

$$
\begin{aligned}
& \phi_{2}=\phi_{1} \in[-\pi, \pi] \\
& \phi_{2}=\phi_{1}+\pi \in[-\pi, \pi],
\end{aligned}
$$

i.e., $T_{u}^{2} \cap T_{d}^{2}$ is the disjoint union of two circles.

Given an $m$-dimensional manifold $Q$, define $H_{k}(Q)$ the $k$ dimensional homology group of $Q$. The dimension of $H_{k}(Q)$ can be interpreted as the number of $k$-dimensional generators of $Q$. For example, for $T^{2}$ it can be shown that $H_{0}\left(T^{2}\right)=\mathbb{R}^{1}$, $H_{1}\left(T^{2}\right)=\mathbb{R}^{2}$ for $T^{2}=S^{1} \times S^{1}$, and $H_{2}\left(T^{2}\right)=\mathbb{R}^{1}$. The geometric interpretation is that the torus has one connected component, two one-dimensional generators $\left(T^{2}=S^{1} \times S^{1}\right)$, and one two-dimensional generator (the space inside the torus). Also, given an $(m-1)$-dimensional closed submanifold $Q_{s}$ of $Q$, the $m$-dimensional relative homology group of $Q$ with respect to $Q_{s}$, defined as $H_{m}\left(Q, Q_{s}\right)$, gives the number of components of $Q-Q_{s}$.

Each contact variety $V_{p_{i}}^{j}$ is a union of two closed curves, each in one torus, with two points in common. However, $V_{p_{i}}^{1}$ and $V_{p_{i}}^{4}$ are common at coincident circles corresponding to the case where all the links are collinear, as are $V_{p_{i}}^{2}$ and $V_{p_{i}}^{3}$. Thus the C-obstacle, $V=\bigcup_{i, j} V_{p_{i}}^{j}$, is the union of $6 n$ circles. We then compute the number of intersections between these $6 n$ circles. Under the general position assumption about the obstacle set $\mathcal{O},{ }^{1}$ we can show that the total number of intersections is $14 n^{2}-11 n$.

Let $\bar{V}$ denote the disjoint union of $6 n$ circles in $V$. The set of intersection points among the $6 n$ circles is denoted $X$. It is obvious that $H_{1}(\bar{V})=\mathbb{R}^{6 n}, H_{0}(\bar{V})=\mathbb{R}^{6 n}, H_{0}(X)=$ $\mathbb{R}^{14 n^{2}-11 n}$, and $H_{0}(V)=\mathbb{R}^{1}$. What is left to compute is $H_{1}(V)$, for which we can use the following exact sequence:

$$
0 \rightarrow H_{1}(\bar{V}) \rightarrow H_{1}(\bar{V}, X) \rightarrow H_{0}(X) \rightarrow H_{0}(\bar{V}) \rightarrow 0
$$

from which we obtain $H_{1}(\bar{V}, X)=H_{1}(V)=\mathbb{R}^{14 n^{2}-11 n}$. Substituting into another exact sequence

$$
0 \rightarrow H_{2}(\mathcal{C}) \rightarrow H_{2}(\mathcal{C}, V) \rightarrow H_{1}(V) \rightarrow H_{1}(\mathcal{C}) \rightarrow 0
$$

yields $H_{2}(\mathcal{C}, V)=\mathbb{R}^{14 n^{2}-11 n}$. This shows that the number of components of the $\mathrm{C}$-free is $14 n^{2}-11 n$.

Since in our algorithm each component is decomposed into at least one cell, the number of cells in a graph with the worstcase number of components is at least $14 n^{2}-11 n$. This gives the asymptotic lower bound of $\Omega\left(n^{2}\right)$. On the other hand, notice that the number of cells in our graph for $\mathrm{C}$-free is

\footnotetext{
${ }^{1}$ No three points are in the same line, no two points and the origin are in the same line, and no two lines each passing through a different pair of points intersect at the same point in the circle centered at the origin and with the radius $l_{1}$.
}

bounded by the product of the number of critical points and the number of collision curves. Let $\delta$ be the degree of the polynomials of collision curves. Then the number of cells in our graph is bounded by $\left(14 n^{2}-11 n+6 n \delta\right) \times(6 n \delta)=O\left(n^{3}\right)$ is the asymptotic upper bound of the worst-case complexity.

\section{CONCLUSION}

This paper presents a method for an exact cell decomposition of the collision-free portion (C-free) of the configuration space (C-space) of a planar closed chain with $m$ links moving among $n$ point obstacles. This method combines the results on the topology of C-spaces of planar closed chains and the decomposition method proposed in [20]. First, the C-space is covered using only two charts. Then, each piece is imbedded in an (m-3)-dimensional torus. The structure of the collision set is used to decompose both tori into cells of collision-free configurations and to construct graphs representing the structure of $\mathrm{C}$-free. The global graph is then established by joining graphs of the two tori if they are connected, as indicated by the analysis of the boundary of the loop closure constraint. A benefit of the decomposition method is that it is very easy to determine cell membership of a given configuration. This fact combined with the exact graph representation of $\mathrm{C}$-free allows one to check path existence by graph search. If a path exists, the cylindrical structure of the free cells facilitates easy path construction.

The proposed algorithm is complete, yet time consuming. In future work, we plan to develop an algorithm that implements an efficient trade-off between sample-based methods and our complete method. Namely, we will refine current sampling approaches so that the sampling density is highest in the regions that are critical to the global topological properties of $\mathrm{C}$-free. These regions are the union of singular loci, which reflect the global structure of the C-space, in particular, the narrow passages. It is expected that by applying refined sampling the computation speed will be enhanced, and the fault probability be reduced.

\section{ACKNOWLEDGMENT}

The authors would like to thank Jim Milgram for introducing them to many of the ideas that led to the results obtained.

This work was supported by the National Science Foundation under grants 0139701 (DMS-FRG), 0413227 (IIS-RCV), and 0420703 (MRI) and by Rensselaer Polytechnic Institute.

\section{REFERENCES}

[1] G. Collins, Quantifier Elimination for Real Closed Fields by Cylindrical Algebraic Decomposition. Proceeding, Second GI Conference on Automata Theory and Formal Languages, Vol. 33, PP. 134-183, 1975, Springer-Verlag.

[2] M. Kapovich and J. Millson, On the moduli spaces of polygons in the Euclidean plane. Journal of Differential Geometry, Vol. 42, PP. 133-164, 1995.

[3] J.-C. Hausmann and A. Knutson, The cohomology ring of polygon spaces. Ann. Inst. Fourier, Vol. 48, PP. 281-321, 1998.

[4] Y. Kamiyama, M. Tezuka, and T. Toma, Homology of the Configuration Spaces of Quasi-Equilateral Polygon Linkages. Transactions of the American Mathematical Society, Vol. 350, No. 12, PP. 4869-4896, 1998. 
[5] J. Hopcroft and G. Wilfong, On the motion of objects in contact. Int. J. Robotics Research, Vol. 4, 1986, PP. 32-46.

[6] J.F. Canny, The Complexity of Robot Motion Planning. Cambridge, MA: MIT Press, 1988.

[7] J.C. Latombe, Robot Motion PLanning. Kluwer Academic Publishers, 1992.

[8] J. Schwartz, J. Hopcroft, and M. Sharir, Planning, Geometry, and Complexity of Robot Motion. Ablex, 1987.

[9] L.E. Kavraki, P. Švestka, J.C. Latombe, and M.H. Overmars, Probablistic Roadmaps for path planning in high-dimensional configuration space. IEEE Transactions on Robotics and Automation, 12(4):566-580, 1996.

[10] S. M. LaValle and J. J. Kuffner, Rapidly-exploring random trees: Progress and prospects. In B. R. Donald, K. M. Lynch, and D. Rus, editors, Algorithmic and Computational Robotics: New Directions, pages 293-308, A K Peters, Wellesley, MA, 2001.

[11] S. R. Lindemann and S. M. LaValle, Current issues in sampling-based motion planning. In P. Dario and R. Chatila, editors, Proc. Eighth Int'l Symp. on Robotics Research. Springer-Verlag, Berlin, 2004. To appear.

[12] J. Yakey, S. M. LaValle, and L. E. Kavraki, Randomized path planning for linkages with closed kinematic chains. IEEE Transactions on Robotics and Automation, 17(6):951-958, December 2001.

[13] J. Cortes, Motion planning algorithms for general closed-chain mechanisms. Doctorat, Institut National Polytechnique, Toulouse, December $16,2003,160$ p.
[14] K.L. Ting. Mobility criteria of single loop n-bar linkages. Trans. ASME, Journal of Mechanisms, Transmissions and Automation in Design, 111(4):504-507, December 1989.

[15] K.L. Ting and Y.W. Liu. Rotatability laws for n-bar kinematic chains and their proof. ASME Journal of Mechanical Design, 113(1):32-39, 1991.

[16] J.T. Schwartz and M. Sharir, On the piano movers II. General techniques for computing topological properties on real algebraic manifolds. Adv. Appl. Math., vol.4, PP. 298-351, 1983.

[17] L. Han and N.M. Amato, A kinematics-based probabilistic roadmap method for closed chain systems. in Algorithmic and Computational Robotics: New Directions, B.R. Donald, K.M. Lynch, and D. Rus, Eds. AK Peters, Wellesley, PP. 233-246, 2001.

[18] J.C. Trinkle and R.J. Milgram, Complete Path Planning for Closed Kinematic Chains with Spherical Joints. International Jounral of Robotics Research, 21(9):773-789, December, 2002.

[19] G.F. Liu, J.C. Trinkle, R.J. Milgram, Complete Path Planning for Planar 2-R Manipulators With Point Obstacles. Proceedings of IEEE International Conference on Robotics and Automation, 3263-3269, 2004.

[20] G.F. Liu, J.C. Trinkle, R.J. Milgram, Complete Path Planning for Planar 3R-Manipulators Among Point Obstacles. To appear in Proceedings of WAFR 2004

[21] D. Halperin, "Algorithmic Motion Planning via Arrangements of Curves and Surfaces", Doctoral Dissertation, Tel-Aviv University, Israel. 